\title{
Pre-cognitive Semantic Information
}

\author{
Orlin Vakarelov
}

the date of receipt and acceptance should be inserted later

\begin{abstract}
This paper addresses one of the fundamental problems of the philosophy of information: How does semantic information emerge within the underlying dynamics of the world? - dynamical semantic information problem. It suggests that the canonical approach to semantic information that defines data before meaning and meaning before use is inadequate for pre-cognitive information media. Instead, we should follow a pragmatic approach to information where one defines the notion of information system as a special kind of purposeful system emerging within the underlying dynamics of the world, and define semantic information as the currency of the system. In this way, systems operating with semantic information can be viewed as patterns in the dynamics - semantic information is a dynamical system phenomenon of highly organized systems. In the
\end{abstract}

This is a pre-print for an article accepted for publication in Knowledge, Technology \& Policy (Springer)

DOI: $10.1007 / \mathrm{s} 12130-010-9109-5$

The publisher's requires that the following note be added:

An author may self-archive an author-created version of his/her article on his/her own website. He/she may also deposit this version on his/her institution's and funder's (funder designated) repository at the funder's request or as a result of a legal obligation, including his/her final version, provided it is not made publicly available until after 12 months of official publication. $\mathrm{He}$ /she may not use the publisher's PDF version which is posted on www.springerlink.com for the purpose of self-archiving or deposit. Furthermore, the author may only post his/her version provided acknowledgement is given to the original source of publication and a link is inserted to the published article on Springer's website. The link must be accompanied by the following text: "The original publication is available at www.springerlink.com"

Orlin Vakarelov

Department of Philosophy,

Social Science Bldg. Rm 213,

University of Arizona,

Tucson, AZ 85721, USA

e-mail: okv@u.arizona.edu simplest information systems the syntax, semantics and pragmatics of the information medium are co-defined. It proposes a new more general theory of information semantics that focuses on the interface role of the information states in the information system - the interface theory of meaning. Finally, with the new framework it addresses the debate between weakly semantic and strongly semantic accounts of information, siding with the strongly semantic view because the pragmatic account developed here is a better generalization of it.

Keywords Semantic information · Pragmatic Information · Dynamical Systems · Information Systems · Precognitive systems · WSI vs. SSI problem

\section{Introduction}

This paper addresses one of the fundamental problems of the philosophy of information: How does semantic information emerge within the underlying dynamics of the world? Let us call this the dynamical semantic information (DSI) problem. DSI is related to problems $\# 2$ and \#4 in the list of 18 fundamental problems of the philosophy of information that Luciano Floridi has compiled $(2010 ; 2004,2008 \mathrm{~b})$. Problem \#2 is The I/O problem: what are the dynamics of information? Problem \#4 is the data grounding problem: how can data acquire their meaning? DSI is also related to a third problem, not explicitly enumerated in Floridi's list. This is the problem of the pragmatics of information: how can information-using systems exist and what makes them such. I tackle three problems of information theory not out of ambition but out of necessity. Indeed, it is one of the central claims of this paper that, in the simplest cases of semantic information, these problems must be approached simultaneously. In the sim- 
plest information systems - those systems that utilize semantic information - the pragmatic, semantic and structural/syntactic aspects of information are codetermined and must be investigated simultaneously.

The paper is related to another kind of problem: can we provide a foundation of cognitive science with the notion of (semantic) information? It is my conviction that we can, and I suggest how elsewhere. (Vakarelov forthcoming) The project of the foundation of cognitive science places a negative constraint on a general theory of semantic information. At least some information systems must be pre-cognitive. Of course, we are cognitive systems, and the kinds of informational media that are interesting for us, such as languages, maps, etc., are allowed to depend on cognitive tools. The gimmicks of cognition make information a powerful phenomenon, no doubt. Still, the project demands, information systems and thus semantic information must be able to exist without cognition.

My strategy for addressing DSI is this: Start with a notion of Information System (IS) that is a special kind of autonomous dynamical system interacting with an environment. Describe semantic information as a "currency" of the information system. That is, treat information for the system not as a primitive but as a derived notion, similar to the way currency is a derived notion of an economic system. Take a decomposition approach to analyzing the components of semantic information - that is, regard notions such as data, meaning, and source, as depicting aspects of informational processes with the information system. Provide a theory of meaning, the interface theory of meaning, for the informational states (data states) of an information medium within the information system.

Finally, I address a current debate about whether truthfulness should be required as a condition for semantic information. The contrast is between a theory of strongly semantic information (SSI), which demands that truthfulness be part of information, and a theory of weakly semantic information (WSI), which regards semantic information as simply meaningful data. My account of semantic information does not fit directly in either of the accounts. It is more general than both. The question is: of which theory is it a more natural generalization, SSI or WSI? I will argue that DSI is a more natural generalization of SSI, and thus it provides indirect support to the SSI view. However, WSI is a theoretically useful notion because it can be interpreted as the "currency" of semantically decoupleable information systems. A unified theory of information, if possible, would require both SSI and WSI notions of information.
The paper is organized as follows: Section 2 introduces the canonical approach to semantic information and points out some difficulties related to the generality of the approach. Section 3 offers an alternative approach suggesting that the most general kind of information is pragmatic information and that semantic information must be investigated within the framework of a pragmatic theory of information. Section 4 introduces the notion of information system and explains why it offers a non-circular basis for defining semantic information. Up to this point the paper develops the conceptual footing of the project. The following sections address the specific problem of DSI. Section 5 outlines the strategy for analyzing information systems, and thus semantic information, as a dynamical system phenomenon - information systems are a special class of organized complex dynamical systems. Section 6 offers a general theory of meaning - the interface theory of meaning - appropriate for the general information systems discussed in the earlier sections. Section 7 addresses the SSI vs. WSI debate.

\section{Canonical Views of Semantic Information}

Few things are canonical about semantic information, but for the purposes of this paper I will assume that canonical views of semantic information have the following form: (1) semantic information $=$ data + meaning (+ truthfulness), (2) the data is conceptually primary. For the moment I will bracket the debate about whether semantic information must be true information. I will return to the issue in Section 7 . Floridi (2003 2010) describes this as the General Definition of semantic Information (GDI). According to this view, to provide an account of information is to provide an account of the structure of the data, and furthermore to provide an account of what makes the data meaningful. The data is a non-empty set of distinctions and each datum is well-formed. The well-formedness condition, minimally, assumes that it is possible to distinguish between the different datums and to separate data from non-data. Some data systems may have further "syntactic" structure. For example, languages may have (compositional) grammar, etc.

Given the data, one can provide an account of how meaning is determined. There can be different "theories of meaning", i.e. different ways to specifying content to a datum. For a language or a map, content may be provided by a reference relation or by specification of a functional role. It is usually assumed that in order for the question of meaning to arise, one must have the system of data on hand. Conceptually, data is more primitive than semantics. Data is, or so the assumption 
goes, a necessary condition for meaning. For, if there are no meaning vehicles, how can we talk about meaning? Meaning of what?

Naturally, semantic information is interesting to an informee because it can be useful to satisfy a goal. Having the information that a tiger is hiding in the bush changes the behavior of the informee to avoid or hunt the tiger. This aspect of information is the problem of the pragmatics of information. It is widely acknowledged that pragmatics places important constraints on semantics and syntax.(Bar-Hillel 1964; Barwise \& Seligman 1997, Dretske 1981; Floridi 2010) Nevertheless, one further, third, assumption of the canonical view of semantic information is that one can provide an account of semantic information independently, and prior to providing an account of how information is used.

Thus, one implicit feature of the canonical view of semantic information is the conceptual priority of data (or syntax) over meaning (or semantics), and of meaning over use (or pragmatics). This idea suggests how to interpret the "+" in the composite expression "data + meaning ( + truthfulness) + use". The " + " is regarded as an amendment operation. Therefore, I will call the view where data is conceptually primary, meaning secondary, and use third-ary, an amendment view of semantic information. The amendment view goes back to Shannon and Weaver, who quite explicitly acknowledge the three levels of problems of information, only to isolate the "syntactic" level, which is the focus of Shannon's theory.(Shannon 1948, Weaver \& Shannon 1963) Carnap and Bar-Hillel (1952) also quite explicitly make the same acknowledgment, only to focus on semantic information. And so do Dretske (1981); Fetzer (2004); Floridi (2003, 2010, 2004, 2005, 2007), and many others, see (Floridi 2010: 3.4) for extensive references.

In many respects, adopting an amendment view is irrelevant to the structure of a formal theory of semantic information. One is interested in describing the structure of the data, the nature of meaning, etc. Which is conceptually prior is lost in the final product. This is important because it implies that even if one questions the amendment view, as I will do in a moment, one does not thereby affect the formal theories of syntactic or semantic information. However, the amendment view affects meta-theoretical judgments.

One important meta-theoretical judgment affected by the amendment view is when one theory of information is more general than another theory. Within the amendment view it is natural to obtain a more general notion of information by obtaining a more general notion of data. Thus, one may start with a paradigm example of an information medium, (e.g.) language, and relax some of its characteristics. One may move from language, to map, to continuous signal systems, to abstract category theoretic system of "classifications" and "info-morphisms".(Barwise \& Seligman 1997) The more abstract and general the theory, the weaker the constraints from semantics or pragmatics.

When is an amendment view appropriate? Obviously, only when the structure of the data can be specified independently. In many cases it can. In formal systems trivially it can. This is partly what makes them formal. Thus, in mathematical theories of information, such as Shannon's probabilistic theory of communication, the related Carnap and Bar-Hillel theory of semantic information, Kolmogorov/Chaitin/Solomonoff algorithmic theory of information ${ }^{1}$ or the Barwise \& Seligman's theory of information flow, etc., one begins with the assumption that the data set is given in advance. A language comes with a fixed alphabet (and a probability distribution); a set of numeric sequences is well-defined (in algorithmic information theory); or, one can define a network of classifications with a fixed set of tokens and types (in Barwise and Seligman's theory). In all these cases one does not provide an account of how the data system is defined, except formally; one simply takes for granted that it is.

But, when can the data set be specified? Formal media, where the data structures can be manipulated independently of meaning or use, can be defined provided underlying stable structures can be created, and mechanisms for reproduction or transformation of the structures can be offered. Purely symbolic media depend on: the stability of the ink on paper, the ability of the human cognitive system to recognize reliably the symbols and how they enter in expressions, the encoding convention of ASCII systems and the ability of devices to copy, transmit, and convert the codes, including converting them to forms readable by a human, etc. Mixed media, like maps or diagrams, have similar requirements. General digital data systems depend on carefully crafted physical devices, such as RAM cells, CPUs, CDs, Hard Drives, etc. All of these devices are crafted to maintain reliable states, to interface with other devices, and to transmit digital signals correctly. That is, the devices meet engineering specifications in virtue of which they count as data and information media. We can safely say that all common information media with clearly specifiable data sets are media that result ultimately of human construction or interpretation.

How about "natural" information media? Are they not independent of human construction or interpreta-

1 For a technical introduction see (Li \& Vitanyi 1997), for a philosophical introduction see \begin{tabular}{r||l||} 
Adriaans 2008 & Grunwald \& Vi-
\end{tabular} tanyi 2008 
tion? We can regard the tree rings, it is claimed, as a data system that contains semantic information about the age of the tree. Note, however, that isolating the rings of a tree as a datum requires ignoring some variations of the tree and focusing on others. The same tree could have had its tree rings slightly differently, yet of the same number and same average thickness. In such a case, the different rings would count as the same data type (similar to the way the letter 'a' can be written with different fonts). Specification of the data system requires specification of what variations are significant and what must be ignored. This idea is captured by the notion of level of abstraction. (Floridi \& Sanders 2004; Floridi 2008a). Specification of a data system always requires a specification of a level of abstraction. In the case of the tree rings, there are nomic dependencies that support the semantic relation between the rings and the age, but the specification of this level of abstraction as opposed to another require $2^{2}$ the interpretation act of a cognitive agent. Nature, in its nomic patterns, offers many opportunities for data systems that can be given semantic significance, it offers ubiquitous potential datums, but it does not offer any well-defined and complete data sets. The tree rings, as a data system, are not constructed by human cognition, but they are interpreted by the human cognition.

It is reasonable to conjecture that the cases where the data set can be specified independently of meaning and use are cases where a cognitive system is involved indispensably in the process of specification. This is not to say that the informational medium must be a part of a cognitive system, or that investigating its informational properties requires bringing in cognition. But, if the conjecture is correct, it would follow that no informational medium appropriate for an amendment analysis could exist without a cognitive system in the background. Now, this is only a conjecture - I have offered only an inductive argument - but it is sufficient to support a methodological prescription: If we want to provide a notion of semantic information that is pre-cognitive, we should reconsider the priority of the components of semantic information.

Instead of starting from within and working outward - by regarding the data set as conceptually primitive - we can start from without and work inward. We can start with pragmatism and let the components of semantic information be co-determined. I call this the decomposition approach, where the notion of semantic information is defined first and then its "components" are decomposed from it as aspects of the informational process.

2 The requirement is not constitutive, of course, but causal.

\section{The pragmatic approach to semantic information}

Pragmatism in general, and the pragmatic approach to semantics in particular, is fundamentally a theory about what the general scenario is where meaning assignment occurs. The general scenario is one where a user utilizes some meaning vehicle system, a medium, in its interaction with another system. In the scenario the meaning, which could be a classical representation relation, is determined by the nature of the interaction and the mode in which the user regards the medium - the significance of the medium for the user in the interaction. The meaning vehicle obtains its semantic characteristics in virtue of the intermediary role that it plays in the interaction. Here I have purposefully avoided some of the technical language used by pragmatist, going back to Peirce, to describe the situation and to provide theories of the sign utilization process - often described as semiosis, following Peirce (1940 based on work from 1890s) and later Morris (1938) ${ }^{3}$ For us the important point is the strategy and its consequences.

One contrast between the pragmatic approach and the canonical/amendment views is what systems are regarded as "more general". For the pragmatic approach the most general scenario is not one that has the most abstract notion of data - where the constraints from semantics and pragmatics are weakest - but one where all dimensions (or parameters) of the scenario are included. The cases where the data system can be isolated and regarded independently of its role in the semiotic process are special cases where an abstraction of the pragmatic and then semantic parameters of the situation is possible. Therefore, the paradigm examples of semantic information media - examples such as languages or road maps - are special, degenerate cases of the general pragmatic scenario. Being degenerate cases, they are atypica ${ }^{4}$, and therefore lessons derived from them should not be regarded as prima facie generalizable.

The classical pragmatic tradition and its more technical offspring, classical semiotics, made similar assumptions about the availability of a mind (or cognition), as the classical analytic tradition motivating the amendment approach. Pierce, for example, readily assumed

3 There are important differences between Peirce and Morris as to the notion of "semiotic", and the structure and purpose for a general theory signs. Most of what nowadays is called semiotics is affected more by Morris' (and Saussure's). (Burch 2010)

4 They are atypical, even though they are ubiquitous and highly salient in human experience. Similarly, an atmosphere high in oxygen is highly atypical as far as planetary atmospheres go, even thought for us it is the stereotypical atmosphere. 
that the user of the signs is a thinking being, and he regarded "ideas" as a sui generis notion in the theory. For him, the mind qua mental, was an irreducible and an essential element of semiosis. The theory was not intended to provide a basis for the study of mind itself, at least not directly.

In the second part of the 20th century, with the emergence of system theory and one of its offspring, cybernetics (Wiener 1965, Turchin 1990), a more abstract, mind-independent version of the pragmatic program was attempted. Within this tradition it became possible to generalize the semiotic notions of sign and sign interpretation with the help of the notion of information. It also became possible to ask whether and how information can be used as a principle of cognition.

The most systematic attempt to understand information within the pragmatic/semiotic tradition was made by Deode Nauta, in his The Meaning of Information. 1970 In it, Nauta argues that information is fundamentally a semiotic notion, and that the most general phenomena where the notion of information is appropriate are pragmatic. That is, in the most general case, the notions of information and information content make sense only when there exists a user of the information and the informational mechanisms are a part of the control mechanisms of the user.

The strategy of pragmatic analysis of information is the following: The most basic notion is information system (or i-system in Nauta's terminology). An information system $s$ is a physical/dynamical system that is in active interaction with an external environment and that satisfies a set of conditions $C$. (We will discuss what $C$ could be below.) The important requirement is that the conditions $C$ do not presuppose the notion of information. Instead, $C$ must be some set of system conditions on $s$ related to the dynamical or physical organization of $s$ and on the mode of interaction of $s$ with the environment. $C$ must guarantee the existence in $s$ of a sub-system, $M$, that can be interpreted as an information medium. Moreover, the functional role of $M$ in $s$ in relation to the interaction with the environment, must be sufficient to define the semantic content of the states of $M$.

According to this strategy, $s$ is an information system not because it operates with meaningful (and truthful) data, i.e. because it operates with information, but conversely, it operates with information because it is an information system. The most important idea is that what counts as data and what gives the data semantic content is determined by the role it plays in the information system.

As stated, all we have is a strategy. There is nothing to demand that the pragmatic approach has some advantages over the classical view. How the strategy performs depends on the conditions $C$, and on how the conditions allow a definition of $M$ and a definition of semantic content. If $C$ is such that one can always define the data set first, and only then the meaning of the data, then the strategy reduces to the amendment view. If $C$ includes some cognitive or mental requirements, then the notion of information cannot be pre-cognitive. $C$, for example, could include the condition that $s$ satisfies the physical symbol system hypothesis ${ }^{5}$ Newell \& Simon 1981) Then, to be a data set is to be a symbolic expression. To have semantic content is to refer to another expression or a symbolic rule governed process. For $s$ to interact with its environment is for $s$ to have some input/output behavior. This example shows that the pragmatic strategy is sufficiently general to capture certain familiar conceptions of information processing. However, if this is all there is to being an information system, the pragmatic strategy is worse off because it brings unnecessary complications. One nice feature of symbol systems is that they can be described formally, only at the level of data relations. There is no point to bringing the user system because it gets abstracted away in the analysis.

The pragmatic strategy offers the hope of producing a condition $C$ that is pre-cognitive, naturalizable, and that clarifies how information systems may emerge. If this is possible, one can use the strategy to provide a naturalistic account of informational phenomena in general. The idea is that it is easier to provide a bottom up naturalistic theory of information systems than to provide a naturalistic theory of information directly. This still does not imply that the amendment view is inappropriate in the general case of information systems. The failure of the amendment view is exposed by the actual proposed solution that fulfills the hope. To this I turn next.

\footnotetext{
5 Newell and Simon offered the physical symbol system hypothesis as a hypothesis about the nature of intelligence: that the collection of intelligent systems is included in the collection of physical symbol systems. In the early days of cognitive science it was common to equate intelligence with cognition. Nowadays, it is mostly recognized that physical symbol systems are not necessary for simpler forms of cognition. Here I am suggesting something stronger, however. I am suggesting that simpler forms of cognition are required for physical symbol systems to exist. This is either because cognition is required for creating physical symbol systems, in the case of artificial systems; or, because a physical symbol system may emerge naturally only within a system that contains simpler cognitive capacities already. I will not argue for this claim here.
} 


\section{Information systems}

Nauta suggests a definition of information systems (isystems). The definition is influenced by ideas form Ackoff (Ackoff 1958) and MacKay (MacKay 1969a). A system $s$ is an i-system when it satisfies the following conditions:

1. $s$ is an open system, i.e. it is a system that is distinct from its environment, but it is in constant interaction with the environment .

2. $s$ is a partially isolated open system, i.e. some of the interactions between $s$ and the environment are structured through well-defined limited channels of influence. Two kinds of channels are significant. One kind is the channels of receptors, $R$, which "transmit" influences from the external environment to the system. Another kind is the channels of emitters (or effectors ${ }^{6}$ ), E, which "transmit" influences from the system to the environment.

3. $s$ is a purposeful system. That is, there is at least one proper set of states, $G$, that the system "attempts" to be in (or near) by affecting its environment. The relation between the behavior of $s$ and $G$ provides the system with normative significance.

4. $s$ contains a sub-system $M$ that can correlate with an external system $S$ (via $R$ ). $M$ can affect the states of $E$. $M$ can be interpreted as a model or a map.

5. $s$ contains a second distinct sub-system (or mechanism) $P$ that filters the states of $M$ and their effect on $E$ to satisfy its purpose. In other words, $P$ steers the system towards $G$ by modulating the effect of $M$ on $E 7$

Let us analyze the conditions of the definition. We will do this incrementally to see what each of the conditions adds. It is helpful to divide the conditions into two groups. Conditions $1-3$ can be regarded as conditions for an autonomous system. These are conditions of the system as a whole. The conditions identify the systems for which the architectural qualification, information system, becomes relevant. Conditions 4 and 5 target the internal organization of the autonomous system that make it an information system - the actual architecture of the system.

First I will focus on the conditions of autonomy. The minimal condition here is the open system condition. A system must be isolated for theoretical focus. It must be possible to identify a sufficiently cohesive,

\footnotetext{
6 Nauta uses the term "emitter". I prefer the term "effector". "Emitter" has the connotation of something being emitted, while "effector" conveys the idea of a general causal effect.

7 We assume that both $M$ and $P$ are non-trivial systems. That is, $M$ and $P$ play an active role in the dynamics of $s$.
}

temporally extended sub-system, $s$, of the world such that description of $s$ in terms of variables restricted to $s$ can be a basis for an effective description of aspects of the behavior of $s$. For example, $s$ may be a rock. The structural links of the molecules of the rock make it appropriate to describe the rock in terms of aggregate variables such as mass, shape, temperature, hardness, etc. When the rock is rolled from a cliff, most of its behavior can be described with such variables and their interactions with external environmental parameters. The effect of the internal molecules is, in a sense, filtered by the system's variables. Similar approach can be adopted to other systems, such as biological cells, animals, robots, etc. General conditions for what makes an arbitrary sub-system a cohesive, isolatable system are complex to provide and are not important for our purpose. The important idea is that there are parameters that apply exclusively to the system; they allow us to talk about the states of the system as distinct from (but not independent of) states of the world. Moreover, the parameters allow us to describe aspects of the dynamics of the system with minimal influence from external parameters. In a cell, many things happen inside that are barely noticeable from the outside, while simultaneously, many things happen outside whose effects on the inside are shielded.

The condition of partial isolation further constrains the class of systems by insisting that some of the interactions between the system and the environment are highly structured. This allows a further state decomposition of the system. Significant aspects of the behavior of the system are determined by the states of the effectors. The set of possible states of the effectors is assumed to be much smaller than the possible states of the system or the possible interaction relations between the system and the environment. (Otherwise, there will be no point of isolating the effectors.) The reduction of states affecting the behavior makes it theoretically useful to describe the system as modulating its behavior. The system is seen as the source of the modulation.

When the receptors are included as another state reduction of the interaction, this time for the influence from the environment to the system, the behavior can be modulated by the control relations between the receptors and the effectors. Significant aspects of the behavior of the system can be described by tracking how the state space of receptors is related to the state space of the effectors. The relation need not be simple. The control relations are modulated by the internal states of the system, including by effects not channeled through the receptors. However, it becomes possible to focus on the internal dynamics of control relations. A small causal/dynamical "pathway" between the receptors and 
effectors acquires high relevance for the behavior of the system. Systematic differences in this pathway are elevated to systematic differences in behavior. We have a phenomenon that we can describe as "high local relevance density".

The last condition of autonomy is the condition of purposeful system. One necessary requirement to describe a system as purposeful is the identification of a set of goal states $G$ and some metric specifying how far the system is from $G$. A second necessary requirement is for the system to have some tendency to move towards $G$ or at least to "put an effort" to move towards $G$. The dynamics of the effectors must be such that it creates a tendency to minimize the distance to $G$, even if external factors completely overwhelm the effort. A fish can swim towards food, even if the current is faster than the fish, and it actually gets farther from the food. Because the condition of purposefulness does not depend only on the system but also on the specification of the set $G$, it is really a relational condition. Every system may be regarded, trivially, as purposeful with respect to some set of states - just make $G$ to be the entire state space. Or, if the system has some attractors in its state space, the set of attractors can make the system into a purposeful system.

Saying that a system is purposeful is interesting if there are independent reasons to identify the set $G$. In some cases such reasons exist. In biological systems natural goal sets are the set of states where the system is in good health, or the set of states where the system is likely to produce fit offspring. More specific goals may be states where the system can have access to nutrients and energy, where it can be protected from predators, etc. What makes such states special may depend on the system's organization. The organization of aerobic bacteria is such that they can extract energy from oxygen. Oxygen has a normative significance for such bacteria, it is a good. (Maturana \& Varela 1980, Weber \& Varela 2002 Di Paolo 2005) If the bacteria can modulate its effectors to move towards an oxygen rich environment, we can regard the set of oxygen rich states as the goal states of the bacteria - its $G$ - and we can regard the bacteria as a purposeful system with respect to $G$. For artificial systems $G$ can be determined by the designer. For the designer of an active seeking missile, the goal states are the states where the missile destroys an enemy.

The idea of autonomy can be summarized as follows: the system can selectively control its behavior so that it can (attempt) to achieve a goal - it can engage in goal-directed behavior. The central idea is that the locus of control is in the system itself, and the control is purposeful. For i-systems, the locus is determined by the higher local relevance density of the receptor to effector pathway, which is part of the system. The goal seeking behavior is determined by the functional role of the effector states with respect to the $G$ states.

This is a minimal notion of autonomy. It is not assumed that the system can represent or internalize the goal. It however focuses the role of the remaining condition of the definition of information system. It sets a problem in a need of a solution. Namely, by what means does the system maintain the control relationship between inputs, outputs and goal? The remaining conditions provide a specific design strategy for this problem: by the means of informational mechanisms. This is important because it means that the pragmatic account of information is not about ends (goals) but about means. Even though information systems, according to Nauta's definition, have functional teleology, the teleological function is not the most important element of the story. As we shall see, it is not what facilitates the information semantics.

The remaining, most important conditions of the definition address the question of means. The body of the definition is the requirement of a sub-system $M$ that is correlated with some other system $S . M$ is the intended system of information vehicles - information medium. It is the system within $s$ whose state space is used for computing the various information measures according to (e.g.) Shannon's mathematical theory of information. The pragmatic approach wants more out of $M$ however. $M$ must play a control role in $s$. The states of $M$ must also be correlated with the states of the effectors, $E$. The correlation between $S$ and $M$ transfers via the correlation between $M$ and $E$ to provide a correlation between $S$ and $E$. Stated in a different way, the correlation between $S$ and $E$ decomposes into two correlations via the mediating role of $M$. It filters the interactions as to facilitate a channel of influence from $S$ to $E$, and thus to modulate the behavior of the system. This is the sense in which $M$ has a control role for $s$. Nauta's story is a bit more complex here, because there is also the intermediary system of receptors, $R$, which presumably mediate between $M$ and $S$. While for modeling sensing systems $R$ is important, for conceptual understanding of the role of $M, R$ is not as important - it even need not be distinct from $M$.

The definition of an information system could have stopped here. All the components for describing various binary information relations are already present. The non-pragmatic approach may even be forced to stop here because what happens after $M$, how $M$ is used in $s$, is not considered defining for a notion of semantic information. The canonical approach attempts to explicate the relation between $M$ and $S$ and to base 
the semantics on this relation. This is for example what Dretske does with the notion of "indicating". (Dretske 1981) This is also how the situation theory group at CSLI approaches the problem 8 The immediate concern that follows is that very simple systems get to be information systems. A thermostat-furnace system can easily be described as a partially isolated open purposeful system with a map $M$ that mediates its behavior. The bi-metal strip is $M$, it correlates with the temperature of the environment (via the temperature of the air around the strip, which can be interpreted as $R$ ) and it turns on or off the switch of the furnace. We can call this the thermostat problem: if a system as simple and silly as a thermostat can count as an information system, then there is something wrong with the notion of information system. One can, of course, bite the bullet and admit that the notion of information is that wide. This is the strategy that Barwise, Perry, Israel, Seligman, and others take.(Barwise \& Perry 1983, Israel \& Perry 1990, Seligmen 1991, Barwise \& Seligman 1997) Or, one can demand some further quality of $M$ that makes the states of $M$ information. One can, for example, add that the states of $M$ are intentional. The problem with this is that it makes the project of naturalizing information systems that much harder. Even pragmatists are not immuned to relying on sui generis mentalist notions. Peirce was aware of the "thermostat problem". He was unhappy with the possibility that sunflowers may be regarded as using signs.(Peirce 1940) His solution was to insist that the interpretant of the sign must be a mind. From a naturalistic standpoint this is unacceptable ${ }^{9}$

A pragmatist approach allows happenings after $M$ to determine its informational status. The proposal requires a further system $P$, a purposeful filter, that mediates the connection between $M$ and $E$. What difference does $P$ make? It has two important effects: (1) it $d e-$ couples $M$ from $E$, and (2) it gives $M$ significance for $s$. The mechanism that implements $P$ modulates the interaction between $M$ and $E$ by controlling how the states of $M$ affect the states of $E$ so that the states of $E$ contribute to (or work towards) moving the system closer to $G$. $P$ contributes additional variables to the interaction so that the states of $M$ only conditionally control the states of $E$. $M$ is not, therefore, merely a causal link in the dynamical interaction, it can be interpreted as a medium. In a description of the system $s$ one can isolate the questions of what the state of $M$ is and what the effect of the state of $M$ is on the rest of

\footnotetext{
8 See in particular (Barwise \& Perry 1983) as well as the many contributions in (Aczel et al. 1993 Cooper et al. 1990 1991)

9 Peirce would have disagreed that the account is not naturalistic. This, however, is related to his somewhat obscure metaphysics, which had pan-physicist elements. (Burch 2010)
}

the system, particularly on $E$. $M$ deserves independent theoretical focus.

The fact that $P$ is a purposeful filter - i.e. it is sensitive to how close the system is to $G$ and it modulates the connection between $M$ and $E$ to minimize the difference - allows the normative distinctions that arise with respect to $G$ to transfer to the states of $M$. The states of $M$ can be evaluated as to their relevance and significance for moving towards the goal 10

Why should i-systems be information systems? We should be careful how we manage our intuitions here. isystems are quite general, and we should not expect to meet the familiar information media immediately. As I insisted above, i-systems are pre-cognitive, while the familiar information media require cognitive machinery. Instead, we should focus on the theoretical merits of describing i-systems as (semantic) information systems. i-systems should be the minimal systems that are usefully modeled as systems receiving information from the world, using the information, qua semantic information, to guide their behavior. It should be accepted that alternative, non-informational models may be available such as dynamical system models or mechanism/causal models - and for some simple i-systems such alternative models may be equally effective in compressing the description of the behavior of the systems.

The question is, why should $M$ be regarded as an information medium? For some, all that is required for semantic information is maintaining sufficient correlation between the situations at $M$ and the situations at $S$. Such correlations are widespread, provided the world contains sufficient patterns and regularities. Why $S$ and why those correlations? To fix $S$ and the appropriate correlations, we need to see what distinctions make a difference, i.e. what correlations are significant for controlling the behavior. But, what are the differences of the behavior that are significant? Those are the differences that "matter". Here the goal states and the goaldirected modulation by $P$ are important. It determines the relevant distinctions which propagate to $M$ and the correlation with $S$. This allows us to isolate the appropriate relations that can be regard as informational.

The decouplability of $M$ from the control system is also important. It makes it appropriate to analyze the system in two stages. First, we can ask: What informa-

\footnotetext{
10 It is not assumed that $P$ operates by "representing" the value of the current state of $M$, and uses the value to select an action. It, of course, may operate like that, but such an operation would likely involve cognitive machinery. In the same way, $P$ should not be assumed to "represent" the goal state and evaluate the action in light of which one would archive the goal under the condition specified my $M$. This would be to regard $P$ as a desire. $P$ is not supposed to be a straight forward generalization of a desire architecture.
} 
tion does the system possess? Second, we can ask: In the circumstances, how can the system use this information? Note that while one can force such a two step analysis to a thermostat, it is pointless because there is no sense in which the thermostat can have the same information and do different things with it. It is more efficient to specify an appropriate equation between the temperature and the state of the switch than to describe the system as utilizing information.

\section{Dynamical Semantic Information}

Over-the-counter modules, such as bi-metal strips (in a thermostat), photo cells, CPUs, etc., make it appear deceptively simple to decompose an artificial (potential) information system into functional units. Its gadgets come with well-defined interface connections, with fixed informationally relevant states, with determined ways they can interact together. Note, for example, that when discussing whether a thermostat is an informational system, it is never difficult to identify what $M$ is - the bi-metal switch - what features of the environment $M$ tracks - temperature - what its function is - to turn on and off the furnace. Similarly with digital computing systems - the input-output states, processing states, and computational operations are all well-defined. Such systems are designed so functional decomposition is easy - in itself not an easy task. Nature, however, does not come so transparently well-structured. It is not easy to determine whether the biochemical network of processes in a bacterium implements an information system, or how (and whether) the chemical and electrical processes in a brain implement an information system.

This is the problem of information from dynamics: How and what organization of the underlying dynamics of the world support the existence of information systems? An account of dynamical semantic information is an account of the conditions for the emergence of information systems within dynamical systems, and what provides the information states with meaning, 11 The word "dynamical" indicates the requirement that the world is viewed as a dynamical system, described with the machinery of dynamical system theory. (Katok \& Hasselblatt 1996. Hinrichsen \& Pritchard 2005) We call this a dynamical system model (DSM) of a system: The world at a given time is described as a point in

11 This is different from (though related to) the question of the information dynamics that some (Williams \& Beer (Forthcoming ) investigate, which is how information propagates and changes within a computational system. a phase space ${ }^{12}$, the space of all possible states of the world. The phase space is usually decomposed into a set of (independent) parameters (a vector base for the space). The temporal change of the world is described as a trajectory through the phase space.

The dynamical system theoretic analysis of a system is essentially counterfactual. One is not only interested in describing the trajectory of the current state of the world. One is interested in describing the dynamical flow of the entire phase space - the trajectories of the world from all possible states. One pays special attention on how the behavior of a system, depicted as a trajectory in the state space, changes with variations of the initial (or current) conditions. Especially interesting are the stability properties of the system - special trajectories, including fixed points, where the system repeats the same states. It turns out that much of the dynamical behavior of the system depends on how the system moves around these special trajectories or points, and how they are distributed throughout the space. This is done in the branch of dynamical systems theory called stability theory. Important ideas here include: (different kinds of) attractors, including the "strange" attractors seen in chaotic systems, basins of stability, bifurcation, chaos, etc. (Hirsch et al. 2004, Jianbo Gao \& Hu 2007, Ivancevic \& Ivancevic 2008)

In a DSM, one often assumes a large all encompassing phase space, global space, but system properties are described with additional phase spaces related to the global space. For example, one may consider only a subset of the independent parameters and describe the behavior of the system in a projection to a subspace spanned by the subset of the parameters (or to a lower dimensional manifold in the space). It could be that the interesting behavior of the system is invariant with respect to some of the parameters, say global position. In this case, by projecting away the invariant parameters, one focuses on the significant parameters. The additional spaces need not be embedded in the large space. They could be related to the parameters in more complex ways. A common simple example is when the system exhibits a periodic behavior with respect to some of the parameters, in which case it may be better to consider a space where only the phase of the period is represented.

An important question in the dynamical description of a system is: What is the smallest (or smaller) set of independent parameters sufficient to describe important aspects of the behavior of a system? We may interpret

12 Often the phase space is called also 'state space'. For example, in quantum mechanics, the Hilbert space of quantum state is called state space. Or, in computer science, the space of possible computation states is called a state space. 
such a question metaphysically as asking what the essential qualities of a system are that determine its behavior. A less metaphysically loaded interpretation is to regard the small set of independent parameters and the equations describing the system's dynamics in the new space as an effective compression of the description of the system's behavior. The possibility of such an effective compression can be interpreted as a discovery of a real pattern in the system. Dennett 1991; Ladyman et al. 2007) When a system is described with a smaller dimensional phase space, and when there is a function mapping the states from the large space to the new space we say that there is a parameter reduction of the system.(Haken 2000; Ivancevic \& Ivancevic 2008) Each parameter reduction defines a partition on the large space where whole sets of state of the large system are regarded as equivalent with respect to the reduced system. Such sets of states can be regarded as macro-states of the system, while in this terminology, the original states in the global system are regarded as micro-states.

In many cases parameter reduction may be appropriate only for a region of the phase space where something of interest happens. Especially interesting cases are when there is some abrupt change in the nature of the trajectory flow of the system. Such changes are described as phase transitions. The classic examples are the state of matter transitions that occur as temperature drops (or increases) that produce change from gas to liquid to solid (or vice versa). Such phase transitions are a more general phenomenon. An important classe of phase transitions are when breaking of symmetry occurs and order increases in the system (as in the transition from a liquid to a solid crystal). Regions where such symmetry breaking happens are especially susceptible to parameter reductions. (Sethna 2009; Ivancevic \& Ivancevic 2008) The new set of reduced parameters is often described as order parameters. (Haken 2000; Ivancevic \& Ivancevic 2008)

Identifying regions of phase transition where parameter reduction is possible can be used to identify (or define) cohesive sub-systems in the global system. If the global system is a chemical reaction system in a fixed spatio-temporal region, the formation of a membrane wall, the emergence of an auto-catalytic cycle or of an autopoietic system, etc., can be identified (in principle) by identifying a region on the phase space bounded by phase transitions, with order parameters tracking the organizational dynamics of the sub-system. The phase transition boundary is the "life/dead" boundary for the system, the internal region is the viability zone, and the order parameters can be the parameters describing the "internal workings' 13 of the system.

Sub-systems can also be nested. In the general case one uses multiple interconnected phase spaces, defined for different overlapping regions of the global space. In this way one can model hierarchically organized systems, and different levels of functional organization.

The global system is regarded as a closed system; when dealing with sub-systems, however, it is important to model the interactions between the sub-systems and their environment. From now on I will drop the 'sub-' prefix and I will assume that we are modeling an open sub-system, consistent with the discussion in the previous sections. Thus, for a system $s$ one separates the order parameters, and the parameters that track external influences - control parameters 14

As a special case of an open system interacting with an environment we can consider an open system interacting with another open system. The interaction can be modeled by additional dynamical equations connecting the order parameters of the two systems. The two interacting systems are viewed as a single coupled system, and the interaction equations are called coupled equations. Of course, the coupled system is itself just a sub-system of the global system, and the coupled dynamics is just part of the global dynamics.

Now that we have outlined some of the key ideas of the DSM model, we want to view information systems as a special kind of sub-systems within a global dynamical system. The goal will not be to provide a general theory of the emergence and dynamics of information systems with DSM. This may still be too hard for the current state of the art of the mathematics of dynamical systems ${ }^{15}$ The goal is to conceptualize information sys-

13 Often the economical order parameters do not have obvious interpretation as degrees of freedom of internal mechanisms of the sub-system.

14 It is important to clear a potential terminological confusion here. The term 'control parameter' is internal to Haken's theory of synergetics. Haken 1993b a Haken calls external influence parameters 'control parameters' because they are often used to control the behavior of self-organized systems. I also talk about control in a less technical sense: I say that a system controls its behavior, or that the locus of control lies in a system. In this more general notion of control, the control relations may depend solely on the order parameters (in Haken's sense) of the system. Also, some control parameters (in Haken's sense) may not have any control significance in my sense. The terminology is unfortunate, but I stick to it to be consistent with the literature. Thus, when the expression 'control parameter' is used, it is always in the technical sense of synergetics. Any other expression that has the word 'control' is used in my (or a control system theoretic (Levine 1996)) sense.

15 There is a large recent literature attempting to analyze informational and cognitive system with the machinery of dynamical systems theory. (Thelen \& Smith 1994 Kelso 1995 van Gelder. 1998 Beer 2000 Chemero 2009 
tems as dynamical systems to be able to view semantic information as a dynamical system phenomenon. We want to focus on what conditions must be met to analyze a system as an information system, described in terms of informational states, semantic relations, and utilization of information.

I will take some shortcuts. I will assume that there exists a dynamical description of the sub-system $s . s$ has a well defined phase space in terms of order parameters. The region of viability $V$ within the global state space is defined so that it is clear under what conditions $s$ exists as a system. A lot of the "internal structure" of $s$ may also be represented in a DSM. An important goal of the dynamical analysis of $s$ is understanding what subset of $V$ has the property that, for a significant period of time, the system will remain within $V{ }^{16}$ In other words, we are interested in the behavior of $s$ from the point of view of its short term survival ${ }^{17}$ The decomposition of the dynamics of $s$ in terms of order and control parameters allows us to analyze the problem as an interaction between the states of the system (order parameters) and the states of the environment that have relevance for the behavior of the system (control parameters). (Some parameters of the global system may be irrelevant for the dynamics of $s$. This may be expressed by saying that there are projections to subspaces (or manifolds) of the global space that preserve the interesting behavior of $s$.) We can examine questions such as: (1) given a fixed state of the order parameters, how will changing the control parameters affect the future behavior of the system, both (1a) as described within the global phase space (or a reduced parameter space that includes the coupling relation with other systems) and (1b) as described in the phase space of the order parameters? In other words, how do the external influences on $s$ affect the behavior of $s$ in relation to other systems and how they affects its internal dynamics? (2) Given a fixed state of the control parameters, how will changes on the order parameters affect the behavior of the system? In other words, how do the peculiarities of the internal organization of the system affect what the system does under the same conditions? Investigating (1) and (2) together allows us to determine where control structures lie. We can isolate to what extent the system's behavior is regulated by the order parameters and their dynamics and to what extent by control parameters, and in what circumstances. We can observe

\footnotetext{
16 Here I am assuming a complex, non-linear dynamics without simple stable regions. There is no trajectory that remains forever in $V$.

17 Essentially, here I am assuming that the goal of the system is survival. Or, stated differently, that the $G$ states in the definition of a purposeful system compose the region $V$.
}

that the behavior is more sensitive to control parameters, in which case the system is more heteronomous, i.e. externally controlled. We can observe that its behavior is more sensitive to order parameters, in which case the system is more autonomous - or, if there is a complex, context sensitive interaction of influences. (3) Given a fixed state of the control parameters, how does changes in the order parameters affect the evolutions of the control parameters? In other words, how do the internal operation of the system affects the external environment, including the external environment that may have an immediate effect back on the system? (4) What are the feedback relations between the control and order parameters?

When examining the loci of control one examines the stability of the dynamical flow of the system in the various phase spaces used to describe it, and the way the stable flow changes with variation of particular parameters. The flow may change continuously, or it may jump discretely from one attractor to another. For example, an automobile's movement is very sensitive to the position of the steering wheel. If one examines how the trajectory of the vehicle changes as the initial position is varied (while the direction is kept fixed ${ }^{18}$ one would observe a parallel flow. As the position of the wheel is changed, the curvature of the flow changes systematically. Note however that the position of the heater dial has no detectable effect on the trajectory. Note also that an explosion of a roadside bomb has a dramatic effect on the trajectory, but variation on the nature of the explosion does not preserve the stability of the flow - the system is not controlled by the explosion even though it is affected by it ${ }^{19}$ In a digital computer the organization of the physical matter is such that fixed sub-systems (memory cells, registers, etc.) exhibit bistable behavior (driven by positive feedback) - i.e. the sub-system orbits one of two stable attractors. In virtue of the fixed organization of the CPU (a kind of fixed constraint on the system - order that does not change) the state of some bi-stable cells can shift (or not) the state of other bi-stable states at a later time (the next clock cycle). If the system has $N$ such cells, it has $2^{N}$ possible attractor states, and at each clock cycle the machine moves from one attractor state to another.

18 Keeping the initial direction is needed for illustrative reasons only. The phase space of the problem is not physical space, but an abstract space including the direction. There is no problem characterizing the stability and variability of the flow with respect to the position of the wheel.

19 Of course, this is relative to the phase space of interest. If the system is parametrized with two states - operative and inoperative - then a bomb can be regarded as a locus of control. It is a binary switch that moves a vehicle from both operative and inoperative states to an inoperative state. 
At each move some cells have control significance, others do not.

While it is possible to localize control significance in many systems, and therefore to place the system in the spectrum between heteronomy and autonomy, such localizations are context dependent and vague. When a car is airborne the control significance of the steering wheel disappears. When the car is on ice the control significance of the steering wheel diminishes and other parameters, such as the position of the gas peddle increase in significance ${ }^{20}$ This is not a failure of the concept of control; it comes from the nature and diversity of dynamical systems.

To capture the notion of information system within a DSM, one must localize control significance to a subsystem $M$ and second order control significance, control over the control role of $M$, to a sub-system $P$. $P$, furthermore, must be evaluated in light of its ability to maintain the system in the viability zone of the global phase space. $M$ must be correlated with a particular set of control parameters - the source system $S$ and have a conditional effect, modulated by $P$, on the general trajectory of $s$ (via $E$ ). The structure and topology of the flow of $s$ admits of such decomposition. There must be an appropriate real pattern of the global dynamics of the system in the viability zone such that one can determine appropriate macro-states (really, a hierarchy of macro-states) and a dynamics of the system respecting the macro-states so that a collection of order parameters exist that track the patterns. Saying that a system is an information system is saying something about what patterns exist within the dynamics that there is a highly structured localized, goal-directed control modulated by a medium $M$.

In the most general case of an information system, the pattern of the dynamics spans the entire system and environment. Even if it is possible to identify the system $M$ independently as a dynamical sub-system of $s$, it cannot be guaranteed that the states of $M$, let us call them the local micro-states ${ }^{21}$ of $M$, are the states relevant for the informational system. We have to fur-

20 On a low friction surface, such as ice, often the only possible way of steering corners fast is using the so called drift method, where the car slides sideways in the direction of the turn and one controls the attitude by adjusting the throttle (gas peddle). This is a very difficult and dangerous technique. Leave it for the professionals! Besides, most modern cars with front wheel drives and electronic stability control cannot drift steer.

$21 M$, of course, is already defined by order parameters, which define macro-state in the global phase space. Indeed, there may be several levels of such macro-states until the right invariance are identified that isolate the system $M$. Still, we can think of the states of $M$ as local micro-states in the immediate reduced phase space of $M$. Yet, further reductions are possible, and further macro-states can be defined. ther identify a collection of macro-states of $M$ that capture the correct distinctions relevant for the information system. It is these macro-states that are interpreted as informational states - as data. More on this below.

Viewing information systems as a special kind of organized dynamical systems allow us to be liberated from some of the proto-mechanistic, incrementalist intuitions that to understand the operation of a system we must understand its parts independently, and then we must recover the system by specifying how the parts fit together. Such intuitions are, I think, at the root of the amendment approach to information. Undoubtedly, in many cases, especially for artificial systems, such an incrementalist approach is the correct one to take. Nevertheless, in many natural systems, such as bio-chemical reaction systems, or neural networks of brains, the incrementalist approach has not been very effective. The dynamical approach to information allows us (in principle) to identify information systems by analyzing the dynamics of the systems - the emerging control relations leading to purposeful behavior. We are still interested in the informational decomposition of the system - in identifying the media and the information relations in which they enter, the data, etc. The language of information systems is not the language of dynamical systems. It is a language - a conceptual framework - that compresses the patterns of interaction in a specific class of dynamical systems in a different way that DSMs.

\section{Interface Theory of Meaning}

I offer a theory of what semantic information is, that unlike the classical view, does not start from a notion of meaning. To be semantic information is to be the currency of an information system. It is still legitimate to ask for a given information state (of $M$ ) $m$ - for a given datum - "What is the meaning of $m$ ?" The decomposition approach demands that an answer to such a question be provided, although it may not look like a familiar answer. In this section I will answer this question in terms of what I call the interface theory of meaning. First, however, I will examine two opposing ways the question is addressed in a friendly crowd. I will use the discussion as a motivation to my proposal.

Traditionally, (foundational) theories of meaning/content, both for language and for mental states, have been divided into two categories: externalist and internalist. There are other interesting divisions, but most are relevant only for sufficiently complex media that require cognitive underpinning. The externalist/internalist division, however is completely general, and can be made for any information medium. Roughly, the distinction is 
about where the primary constraint of the determination of "meaning" for an information state derives from. An externalist theory focuses on constraints outside of the user of the informational state. Particularly, it focuses on the relation between the informational state and the sources or object of the information. The meat of the semantic connection derives from some nomic (or teleonomic) connection between the source system and the information medium (receiver) system. The focus of semantics for an externalist theory is the determination of the way the world is. Examples of such theories are Dretske's and those of the situated semantics group. Also, Millikan's and those of the teleosemantic community. Millikan 1987, 1995, 2006) I will focus below on an account due to Bogdan (Bogdan 1988a, 1994), that is quite similar in spirit to mine. Bogdan's account differs from other externalist in that it takes goal-directedness to be a fundamental requirement for semantic information.

An internalist theory, on the other hand, considers as the primary constraint of meaning what the information state does for the user. The model of the internalist account is not reference fixation and fact determination, but message interpretation. The question that an internalist asks is not what $m$ means, but what $m$ means to a given user. Of course, for $m$ to be informative about the world, it better be sufficiently correlated with a source, but this is not a constitutive condition of the meaning of $m$. It is a condition of a good interpretation system. In an internalist account, $m$ can have a meaning for a user even if the user is, so to say, completely out of it. Below I will consider the internalist account of MacKay (1969b; 1969c), who, as I indicated above, was a strong influence to Nauta.

Bogdan, like me, wants a notion of semantic information to serve as a basis of understanding cognition. He makes a distinction between material information and semantic information. Material information in one receiver system from another source system results from the systematic, nomic relation between structures of the source and receiver. Such a notion of material information is fairly uncontroversial among people that take the notion of information seriously. It is what some describe as environmental information (Floridi 2003; Barwise \& Seligman 1997, Dretske 1981), or physical information, or potential-information (Nauta 1970). Semantic information is a kind of material information where the "from" is converted to "about" - it is when the receiver can be said to have information about the source. One way of understanding Bogdan's effort is as explicating in a naturalistic setup the notion of aboutness. To this end, semantic information is characterized as follows: "Semantic information is material informa- tion with a functional business determined by teleology." (Bogdan 1988a 89)

The key task is explicating the notion of "functional business determined by teleology". Bogdan's theory is complicated and it is not my goal to develop it here, nor is it to compare it to my use of goal-directedness which is based on Nauta. There are many similarities, and some apparent differences in the two approaches, but, I must admit, it is still not completely clear to me how deep the differences go. Here are some ideas that cast light on how teleology "converts" material information into semantic information. It does this in at least three ways: (1) the goal acts as a filter of relevance for aspects of the information source. Only some of the aspects (or features) of the source are relevant for goal-directed behavior. (2) It determines internal, architectural functions for the system using the material information to achieve the goal. It is not sufficient for the system to have material information from the source of the aspects relevant for the goal. The system must be organized in a way material information which is nothing more than a form of nomic correlation - can affect the system's goal-directed behavior. (3) It solves the proximal stimulus problem, i.e. it allows distinction between the true source of the information and any proximal systems in the information pathway from the source to the receptor that co-vary with the source. For example, a state of a visual system may contain material information from a chair, but also it may contain material information from the retina. Only the chair is relevant to the goal-directed activity of finding a place to rest. The retinal state has no rest-inducing properties.

(1) and (2) provide a basis for a system to utilize semantic information - to select (and convert) material information to meaningful information. There is similarity between Bogdan's conditions (1) and (2) and Nauta's conditions of i-system. It is condition (3), however, that separates from from about. It fixes the content of the information.

The semantics of Bogdan's notion of semantic information, the answer to the question "What is the meaning of an information state $m$ ?", is of the form: $m$ is about a system $s$ and it is in so-and-so state (or has so-and-so probability distribution) ${ }^{22}$ The back-end of the information process - the functional business focuses (or constrains) the front end - the correlation

22 Bogdan actually does not address this question in Bogdan 1988a 1994). He resists applying the term 'meaning' to such simple systems to avoid undesirable connotations. (Private corresponds) But it seems to follow from the discussion that this would be the form of the answer, if one must be given. 
part - but it is the correlation that determines the meaning.

Now let us consider an internalist theory. MacKay's internalist account of meaning is aimed for the following general situation. There is a system $S$ that is capable of goal-directed activity. $S$ receives a "message" $m$. $m$ could be a message sent by another system with a specific intention, or it could be a signal from the environment. What does $m$ mean for $S$ ? He proposes the following answer:

"[T]he meaning of a message can be defined very simply as its selective function on the range of the recipient's states of conditional readiness for goal-directed activity." (MacKay 1969b 24)

Consider the following example, adapted from MacKay: Steve is seating on an armchair reading a book. Somebody enters and says "It is raining." (This is $m$ ) Steve does not make any differential response to the statement. What has $m$ done? It has not changed the behavior of Steve in any way (assuming that he continues to attend to the book with full engagement). However, when Steve stops reading the book, as he leaves the room he grabs the umbrella. The message has induced in Steve the readiness to take the umbrella, or to imagine the streets wet, or to be concerned about whether he brought the lawn mower to the garage, etc. Steve (read his cognitive system) is in a conditional state of readiness to respond differentially to various states of his environment. The message "it is raining" changes these conditional states. Metaphorically speaking (MacKay's metaphor) the message adjusts the switch-boxes of Steve's response function. It is this tendency of a message to select the switches of the response function that determines what the message means for Steve. The same message, qua physical (syntactic) form, would adjust the switch-box of Peter in slightly different ways. It, therefore, would have a different meaning for Peter. The message would normally change the conditional state of readiness of both Steve and Peter in similar ways, allowing them to coordinate their actions. If Steve calls Peter for a ride from work Peter would not be surprised, and Steve would expect Peter not to be surprised, etc.

According to MacKay, it does not make sense to ask about the meaning of $m$ in isolation - the notion of meaning makes sense only relative to a user. Meaning does not dependent only on the form of the message. Of course, for some classes of users, messages of particular forms change their conditional state of readiness systematically. This may be so because of fixed conventions or because the form of the message has a useful correlation with the environment in which the goal di- rected activity takes place. Communication in a common language would be impossible otherwise.

It is easy to take the externalist and internalist approaches to the question of the meaning of $m$ as incompatible. They, after all, have very different form. The externalist approach excludes the user from having a constitutive role for meaning. The internalist approach makes meaning primarily user dependent. The approaches need not be incompatible however. One strategy for reconciling externalism and internalism is to take a hybrid account of meaning/content. Such hybrid theories are motivated by an observation that external or internal considerations are not sufficiently fine grained. Such hybrid views are especially important in discussion of mental content. Putnam's Twin-Earth thought experiment (Putnam 1975) can be interpreted as suggesting that a purely solipsistic internalist theory of mental content would not be able to distinguish between the different contents of water (twater) of believers among Earth and Twin-Earth inhabitants - or so the intuition goes. Such hybrid theories of meaning have targeted cognitive information media - languages, mental states (beliefs), etc. This analysis of meaning cannot easily transfer to the domain of $\mathrm{dy}$ namical semantic information.

In the case of dynamical semantic information, the externalist and internalist conceptions of meaning collapse into a single notion. The reason for this is the codetermination of macro-state structure of informational systems. Let us examine this in a bit more detail. As indicated above, to claim that a given open dynamical system is an information system is to identify a collection of sub-systems, a collection of macro-states for each sub-system, and a set of dynamical relations that respect the macro states (plus various other things). Let us focus on the macro-states. For each sub-system, e.g. $S$ or $M$, we must determine what micro-states should be regarded as functionally equivalent with respect to the dynamics of the information system, qua information system. In the case of $M$, this amounts to specifying what the data states of the system are. In the case of $S$, this amounts to specifying what the structure of the source looks like form the prospective of the information system?23 It is possible that both $S$ and $M$ have independent macro-structure and the dynamical correlation relevant for the information system matches this independent structure. $S$ is what it is intrinsically, $M$ is what it is intrinsically, and they are simply connected by some causal process that matches the properties of $S$

23 This may be the informational equivalent to (and the dynamical basis of) the phenomenological notion of umwelt (von Uexküll 190919321982 . Nauta explicitly utilizes the notion in his analysis of information systems. 
with the properties of $M$ and thus an information connection is formed. This possibility is, in fact, the standard conception of the information process as physical phenomena. While such a dynamical scenario is possible, it is not necessary.

In the general case we cannot assume that the macrostructure relevant for the information system description is determined "locally". We may need to look at the entire dynamical system - the entire process of interaction between the system and the environment - as the basis of determination of the macroscopic structure ${ }^{24}$ To determine whether a particular macro-state of $S$ is informationally relevant, i.e. whether it is differentially significant for the purposeful behavior of the system, we must trace the dynamical trajectories of the system and determine (at least) two things: (1) whether the microstate variation within the macro-states is insignificant for the purposeful behavior, i.e. the dynamical trajectories in the appropriate reduced phase spaces that track the viability parameters (or in general the parameters related to the goals states) are stable. Let us call such macro-states informationally stable. (2) Whether other informationally stable macro-states produce a differential dynamical response of the system in the same reduced phase space.

Not all informationally relevant states of $S$ need make a difference for the system qua information system. Only in the distinctions that make a difference (MacKay 1969a) for the internal control mechanisms mediated through $M$ and modulated by $P$ are important. In other words, only some of the informationally relevant states are actually significant for the system.25 What states of $S$ are significant depends on the internal organization of the $M-P$ system. The metabolism of a bacterium may respond differentially to many types of nutrients. This is significant for the goal state of the system. Some nutrients may be better at maintaining the

${ }^{24}$ It is very temping to describe the problem with the language of supervenience. I recommend caution in using supervenience here because the notion of supervenience has its roots in the classical object/property metaphysics, while my discussion is based on the dynamical system theory approach to system analysis. The two approaches are not incompatible, although I think that the dynamical systems approach is more general. The notion of supervenience, as used by Kim for example, is not readily convertible to the dynamical systems approach. But, if I must describe the problem in terms of supervenience, I can describe it as follows: we cannot assume that the macro-properties of the sub-systems supervene on the micro-properties of the same subsystem. They may supervene on the entire environment/information system ensemble.

${ }^{25}$ In fact, only a small subset of the informationally relevant states of $S$ would, in general, be significant for the internal control pathway. This difference - a kind of informational deficiency of the system - is central for understating the role of cognition for an organism. (Vakarelov forthcoming system deep into its viability zone; others may merely slow down the exit ${ }^{26}$ Still, the control mechanisms that modulate the bacterial purpose-modulated response to the nutrients (if they exist) may make only a small number of distinctions. Even more - the internal dynamics may force and utilize distinctions that, considering $S$ as an independent system, are not derived from the structure of $S{ }^{27}$ It follows then that in the general case, we cannot assume that the distinctions in $S$ that are relevant for the semantic evaluation of a datum depend on $S$ alone. They depend on the dynamics of the entire system. From the prospective of the information system, there is no independent "objective reality" that its informational media track. This does not preclude the possibility that an external observer can identify both independent (from the information system) real patterns (or properties) of $S$ and real patterns emerging from the informational interaction.

Nor can we assume that the informational states of $M$ be specified independently of $S$ and the rest of the system. The appropriate macro-state structure of $M$ is, in general, under-specified by the correlation to $S$ alone, or by the relation to the effectors $E$ of the system alone, or by the effective distinctions that $P$ can make alone. Indeed, there can be structural relations between $S$ and $M$ that could be useful for the goal directed behavior of the system but that never make it to control service. Similarly, there can be many interesting connections between $M$ and $E$ that could be relevant for behavior but the corresponding macro-states of $M$ may not track anything interesting in the environment, etc. Again, in the general case, the entire dynamical system/environment complex must be used to identify what macro-states of $M$ count as the data states of the system.

Of course, it does not follow that one always must consider the entire complex. There can be sufficient internal structure to $S$ or $M$ or $P$ or $E$ so that the significant macro-states stand out on their own. Many artificial information systems may be designed with highly structured components that fix the relevant macro-states from within. Evolutionary processes may also generate highly modular systems where the relevant macrostates are fixed from within. Still, a general theory of

26 Eating spoiled food may help an organism not die of hunger now, but it may cause food poisoning that may harm the organism later.

27 Some (Maturana \& Varela 1980 Varela et al. 1992 have suggested that the system of color discrimination and categorization of many organisms only partially depict physical reflectance (or other) properties of external objects. It depends to a large degree on the internal dynamics of the visual system. In a sense, the organism imposes structure on the world that is not there independently, but that is utilized by the system. 
semantic information must admit information systems where all the relevant patterns of organization derive from the entire complex - no off the shelf parts.

It follows that neither an external relation between $M$ and $S$, nor an internal function of "selecting conditional readiness states" is sufficient to provide a general notion of meaning, for they don't even fix the syntax of the information system independently. To specify the meaning of a state $m$ we must do something different.

What does $M$ really do in the information system? It acts as an interface between the (external) world and the control system. It structures influences to allow focused purposeful control. If any sense of significance can be given to a particular state $m$ of $M$, it must be related to this interface function. The significance of $m$ is neither that it tracks something external nor that it can affect the control mechanisms of the system, but that it can connect one to the other. The idea of interface allows us to specify a notion of meaning for a datum in an information system:

Meaning: The meaning of an informational state $m$ of an information system is given by the differential interface function it serves in the whole process of purposeful interaction between the information system and the environment.

In what sense is this a definition of meaning? This is clearly not a definition produced by conceptual analysis of "meaning". It is not intended to explicate or capture typical "meaning ascriptions". Almost exclusively, meaning (or content) ascriptions involve languages or language-like entities like beliefs. As noted above, within the pragmatic approach of information such media should not be seen as stereotypical. In fact, they should be seen as atypical. I would regard a general account of meaning that looks too much like an account of meaning for language as suspicious.

My definition is foremost technical. Still, there has to be some connection between it and more common notions of meaning. Otherwise it makes a deceptive use of terminology. The proper connection is that of generalization. The way to evaluate whether the defined notion deserves to be called "meaning" is to satisfy the following two requirements: (1) It must be a notion that can be defined within appropriate general framework. (2) When the general framework is instantiated to the stereotypical case(s), the notion must "reduce" to the traditional notion. Thus, if the medium of an information system is a language, then the differential interface function related to the linguistic expressions must boil down to something like a stereotypical notion of meaning. It must be noted that an instantiation operation, as an inverse to a generalization operation, is one-to- many $2^{28}$ Generalization always looses complexity, so a language-using information system may involve complexities that do not appear in the general case and that may produce different notions of meaning depending on how the complexities are fixed ${ }^{29}$

Most of the work in sections 4 and 5 was done to assure us that nothing irreducibly semantic lurks in the notion of information system viewed as a dynamical phenomenon 30 Therefore, (1) can be achieved. (2) is much more difficult. It requires the specification of an information system that utilizes language semantically. This is a daunting task. Current states of psychology or computational linguistics are very far from understanding how languages can emerge in natural biological agents or how to design artificial agents that use semantic language. The kind of informational systems that may get anywhere close to being language users are vastly more complicated than the simple precognitive information systems discussed here. It follows that providing a full justification of the interface function conception of meaning as a proper generalization of stereotypical conceptions of meaning is beyond my current abilities. What I offer is a conjecture that the interface theory can contribute towards understanding of semantic processes for language-using or other cognitive information systems. I can provide however some

28 Of course, the opposite is true too. There may be many ways one can generalize a specific situation. Here I follow one specific generalization supported by the pragmatic approach.

29 The theoretical method of generalization and re-instantiation is a great tool for resolving disagreement between competing theories of something (e.g. of meaning). By obtaining a general theory and then showing how specific but competing scenarios are instances of the generalization, one can demonstrate that the disagreement is not conceptual but results from a different fixation of some theoretical parameters. It may turn out that both specific theories are correct but they are theories for different domains, and moreover, both are justified in using the same concept because the concept turns out to be a specific instance of the general concept.

30 There is one important contention here. Isn't the notion of goal, and thus purposeful system already semantic? Such an objection has been raised by Dretske $(1988)$ in response to Bogdan, and more generally by Floridi $(2010)$. Careless use of goals can indeed sneak in semantics. The important thing is not to assume that goals are explicit (like desires). Goals should not be regarded as kinds of propositional attitudes. My, and I believe Bogdan's, notion of goal is not content determining. For Bogdan's reply to Dretske see (Bogdan 1988b). In my case, the notion of purposeful system is purely dynamical. It captures a particular patterns of interaction between a system and its environment. Such a pattern may be selected by an external designer, in which case Floridi's zero semantic condition (Floridi \& Taddeo 2005 2007) is not satisfied, but it could result from (or be) a natural pattern in the global dynamics. As it has been argued by some (Maturana \& Varela 1980, Varela 2000, Weber \& Varela 2002), convincingly at least to me, the phenomenon of life may be related to the natural emergence of purposeful systems. This, however is a separate issue that I do not wish to discuss here. 
hints about how the notion may lead us towards more familiar notions of meaning.

Let us go back to the observation that the definition collapses the external and internal conception of meaning. Specifying the differential interface function of a state requires looking at the entire system/environment complex. We can think of the datum state $m$ as participating in a process ${ }^{31}$ of interaction where causal effects from the environment are channeled through the internal M-P control pathway to produce actions, which actions modify the system's behavior, and which in turn changes the state of the environment (including the relations between the system and other external systems). This affects future causal effects and the way the system responds to them. The complex feedback process can be decomposed into segments/sub-processes. It is possible to focus on the regularities that exist between the system $M$ and the source $S$, or on the regularities that exist in the way a datum affects the state of conditional readiness of the system. In other words, it may be possible to extract two interface sub-functions, one related to the external correlation between the medium and the source, and another related to the selective control function of the medium. It is not clear that the interface function is always completely determined by the two sub-functions, but it is important that we can recover the external and internal notions of meaning as aspects of the process.

The story gets more interesting when the structure of $M$ and $P$ gets more complicated - particularly, when the system utilizes different sub-systems that act as information media. The system may have media $M_{1}$, $M_{2}, \ldots M_{n}$ (and a collection of different purposeful filters), each with different roles and interface connections. Some media may be connected to different external systems or different aspects of the same systems, others may interface with other media, yet others may be connected with effectors or control the states of other media, etc. When the system is organized as a complex network of information media, complex interface (sub)functions can emerge. Some can depend almost exclusively on external connections to outside sources, others can be analyzed entirely in terms of their control role or effects on other media. I conjecture that the canonical examples of information media that shape many of our intuition about semantics are media that exist (within an information system) as only one of a large network of other information media that jointly control the system's behavior. Thus, to take correspondence theories of meaning as an example, it is tempting to say that the word 'chair' means a property of external objects.

31 Here I use the notion of process informally. It is assumed that the system is ultimately describable with a DSM.
Thus, in the expression, "This is a chair," the meaning is given by some fact in the world that the object depicted by the indexical has the property of chairhood. In an information system using language we can analyze this idea in a different way. The language medium, whose datum may be some structural equivalent to the expression "This is a chair," interacts with other nonlinguistic media connected to perception, allowing the system to identify and interact with patterns in the world that can be clustered through some data state of some internal media. To make Fodor happy, we can assume that there is a single medium that gets in an information state uniquely correlated with chairhood a kind of a concept of "chair". The language system, in this picture, is not interfaced with the world (or some abstract realm of propositions). It is interfaced with other information media. The properties of the interface relations look a lot like the properties that a correspondence semantics may have, but these interface relations do not capture the true interface roles of the language datums for the information system. To determine the true interface role, we need to link all local interfaces and see how the entire complex participates in the purposeful behavior. In other words, the correspondence relation underlying correspondence semantics, according to my rough hypothetical pragmatic analysis, is a relation that exists only between carefully orchestrated media, not a relation that exists between the datum and the world. However, such an inter-media information relation can be elevated to important cognitive significance by the other media and their interfaces with perception and action.

In this picture there is no need for some mysterious sui generis mentalist notion of intentionality (or Pierce's notion of interpretant) to support the semantics; it is all a story of organized dynamical sub-systems of $s$ channeling and controlling effect of external and internal influences on behavior. It is all complex patterns in the dynamics of the world, patterns of the flow of the global phase space.

\section{SSI vs. WSI}

I will end with a short discussion of how the notion of dynamical semantic information relates to the debate about whether truthfulness must be included as a condition of semantic information. One position states that information is simply meaningful data. This is the so called weakly semantic theory of information, (WSI). Proponents of WSI are (Carnap \& Bar-Hillel 1952, Fet- 
zer 2004) 32 Another position insists that truthfulness must be included in the definition of information. This is the so called strongly semantic theory of information, (SSI). Proponents include Dretske (1981); Barwise \& Seligman (1997); Floridi (2010). The most systematic defense of SSI can be found in Floridi (2004, 2007, 2010). The gist of the debate is the following: imagine Steve receives a message "It is raining outside". When has Steve received a piece of information? A proponent of WSI claims that Steve has received a piece of information regardless of whether it actually is raining outside. A proponent of SSI claims that Steve receives information only if the statement is true, i.e. only if it is actually raining outside. Another way of formulating the debate is whether mis-information (when it is not raining) is a kind of information or a kind of noninformation (a kind of pseudo-information). For WSI mis-information is a kind of information. For SSI misinformation is something different all together. (To use Dretske's metaphor, mis-information is no more a kind of information that a rubber duck is a kind of duck.) My goal here is not to enter the debate head on, but to ask a different question: To which notion of information is DSI a more appropriate generalization?

Floridi is clear that the question about whether truthtfulness is necessary for information is specifically targeted to declarative, factual semantic information.(Floridi 2010) This is the kind of informational presentation where one can separate the question of meaning from the question of truthfulness. Let $d$ be a datum. One asks two separate questions: (1) what is the meaning of $d$ ? (2) Is the fact depicted by $d$ the case? It should be clear immediately from the discussion in the previous sections that in DSI we cannot assume that a datum can have meaning independent of the way it partakes in the dynamical process of a user interacting with another system $S$. In fact, in the general case, what the datum is is determined by the entire process (including counterfactual conditions). From the point of view of DSI, the case of declarative information for which the questions (1) and (2) can be separated is a restricted case of semantic information. I would claim (though I would not argue for this here) that deliberative information is a cognitive phenomenon.

Which theory, WSI or SSI, needs the separation more? WSI cannot even be formulated with media for which questions (1) and (2) cannot be asked separately. To say that meaning, but not truthfulness, is necessary for a datum to count as information demands specifying the meaning of the datum independent of the circumstances under which it is truthful. SSI does not require

32 ... and most everyone working in the field of IST. See $\sqrt{\text { Floridi }}$ 2010 4.2) for many examples and references. the separation, even though for declarative information it is formulated with the separation at hand. If one takes the pragmatic approach to information, SSI appears to be a more general theory. Now, this may sound counter intuitive. WSI appears to be more admissive than SSI - it allows for more things to count as information. SSI, after all, places a further constraint on the condition of information - it adds a condition of truthfulness. WSI says: information $=$ data + meaning. SSI says: information $=$ data + meaning + truthfulness. The intuition that WSI is a more general notion of information is a consequence of the amendment view of information. The pragmatic approach however measures generality in a different way. SSI is more general because it supports a conception of information that is applicable to a wider set of systems. Let us see why!

According to DSI, in the most general case, the structure of the data, its differential interface function in the system (its meaning), the informationally relevant distinctions in the source, and the available effector states (the possible informationally controlled actions), are determined by the global pattern of interaction. The structure of the data and its meaning cannot always exist as distinguishable macro-states without the interaction with $S$ and the purpose modulated control relation between the information medium and the effectors. Using the notion of truthfulness in the situation is perhaps an undesirable stretch of terminology - it should be reserved for declarative information - but the informational states in the system are significant because of the way they are actually correlated to the states of the world and because of the way they control the behavior of the system in light of those correlations. This is, I think, the true motivation behind the SSI. The insistence for the condition of truthfulness was never motivated by a conviction that a formal semantic valuation must be added to the concept of information. It is not about merely fixing an alethic parameter to 'true'. The motivation behind the theory has always been the idea that when we say that someone has information about something we are interested in how the state of the world is internalized in the person (or organism, robot, etc.) and how the person can act accordingly. When one asks for information about the weather one does not ask for a random meaningful statement with the weather as the topic; one asks for a link to the weather so that she can change her actions accordingly. In a sense, the motivation behind SSI has always been (a hidden form of) pragmatism.

Couldn't one object, however, that my analysis of WSI is unfair? It could be claimed that DSI generalizes WSI just as much as SSI. The two theories simply collapse when one cannot separate between questions (1) 
and (2). Indeed, if we can talk about data, as DSI does, and talk about meaning, as DSI does, then WSI can call it information. This is a valid response and a possible theoretical choice, but I think that is a wrong choice. The reason is that it violates the spirit of WSI. The reason WSI proponents want to call more things, false things, information is because they think that for many problems related to manipulation of information ${ }^{33}$ the truthfulness value of the data states is irrelevant. One may want to study how a person, a robot, or an expert system manipulates informational states to, say, develop a theory of reasoning. For many theoretical problems related to information systems (in the IST sense) the truthfulness value of the informational states may be ignored. For such problems it is important to have a theoretical notion that maintains alethic neutrality.(Floridi 2003, 2010) Such problems can be important for pre-cognitive information systems (in my sense) as well. The pragmatic strategy approaches the problem of information by starting with the notion of information system and then defining information as a currency of the system. This means that a theory of information systems (in my sense) must be able to investigate the internal informational dynamics of the system - any invariance and patterns that exist. It would be required to have a theoretical notion that abstracts away from some of the constitutive elements, such as the source, which determine the structure of the information system. The approach of WSI provides exactly this. It provides an abstract theoretic notion that can be used for investigation of the "currency" information, qua internal system operation. It should be distinguished from the more general notion of information related to the highly structured dynamics of a system interacting with an environment.

The debate between WSI and SSI is, therefore, a bit misguided, in the sense that both ideas are theoretically important and a mature general theory of information should have use for both. I would prefer if the term 'information' be preserved for the more general notion connected with SSI, and another term is used for the abstract notion of WSI, but I would live with the abusive notation of calling both 'information', as long as the context makes it clear whether the alethic/pragmatic dimension has been abstracted away.

\section{References}

Ackoff, R. L. 1958. Towards a Behavioral Theory of Communication. Management Science, 4(3), 218-

33 ...and not merely manipulation of data. In information systems theory (IST) one is often interested in manipulating information in a semantically sensitive way.
234.

Aczel, P., Israel, D. J., Katagiri, Y., \& Peters, S. (eds). 1993. Situation theory and its applications. Vol. 3. CSLI.

Adriaans, P. 2008. Philosophy of Information: Concepts and History. In: Adriaans, P., \& van Benthem, J. (eds), Handbook on the Philosophy of Information. North Holland.

Bar-Hillel, Y. 1964. Language and Information: Selected Essays on Their Theory and Application. Addison Wesley.

Barwise, J., \& Seligman, J. 1997. Information Flow: The Logic of Distributed Systems. Cambridge Tracks in Theoretical Computer Science, no. 44. Cambridge University Press.

Barwise, J., \& Perry, J. 1983. Situations and Attitudes. Bradford Book.

Beer, R. 2000. Dynamical approaches to cognitive science. Trends in Cognitive Sciences, 4, 91-99.

Bogdan, R. J. 1988a. Information and Semantic Cognition: An Ontological Account. Mind and Language, 3(2), 81-122.

Bogdan, R. J. 1988b. Replies to Commentators. Mind and Language, $\mathbf{3}(2)$, 145-151.

Bogdan, R. J. 1994. Grounds for Cognition: How GoalGuided Behavior Shapes the Mind. Lawrence Erlbaum Associates.

Burch, R. 2010. Charles Sanders Peirce. In: Zalta, E. N. (ed), The Stanford Encyclopedia of Philosophy (Spring 2010 Edition).

Carnap, R., \& Bar-Hillel, Y. 1952. An Outline of a Theory of Semantic Information. Tech. rept. 247. MIT.

Chemero, A. 2009. Radical Embodied Cognitive Science. MIT Press.

Cooper, R., Mukai, K., \& Perry, J. (eds). 1990. Situation Theory and its Applications. Vol. 1. CSLI.

Cooper, R., Mukai, K., Barwise, J., \& Perry, J. (eds). 1991. Situation theory and its applications. Vol. 2. CSLI.

Dennett, D. C. 1991. Real Patterns. Journal of Philosophy, LXXXVIII, 7-51.

Di Paolo, E. A. 2005. Autopoiesis, Adaptivity, Teleology, Agency. Phenomenology and the Cognitive Sciences, 4, 429-452.

Dretske, F. 1981. Knowledge and the Flow of Information. MIT Press.

Dretske, F. 1988. Commentary: Bogdan on Information. Mind and Language, 3(2), 141-144.

Fetzer, J. H. 2004. Information: Does it Have To Be True? Minds and Machines, 14, 223-229.

Floridi, L. 2003. Information. In: Floridi, L. (ed), The Blackwell Guide to the Philosophy of Computing and 
Information. Oxford - New York: Blackwell.

Floridi, L., \& Sanders, J. 2004. Levellism and the Method of Abstraction. IEG Research Report IEGRR-4. Oxford University.

Floridi, L. 2004. Outline of a Theory of Strongly Semantic Information. Minds and Machines, 14(2), 197222.

Floridi, L. 2005. Is Information Meaningful Data? Philosophy and Phenomenological Research, 70(2), 351370.

Floridi, L. 2007. In defence of the veridical nature of semantic information. European Journal of Analytic Philosophy, 3(1), 31-41.

Floridi, L. 2008a. The Method of Levels of Abstraction. Minds and Machines, 18(3), 303-329.

Floridi, L. 2008b. Trends in the Philosophy of Information. Pages 113-132 of: Adriaans, P., \& van Benthem, J. (eds), Handbook of Philosophy of Information. North Holland.

Floridi, L. 2010. The Philosophy of Information. Oxford.

Floridi, L., \& Taddeo, M. 2005. The Symbol Grounding Problem: a Critical Review of Fifteen Years of Research. Journal of Experimental and Theoretical Artificial Intelligence, 17(4), 419 - 445.

Floridi, L., \& Taddeo, M. 2007. A Praxical Solution of the Symbol Grounding Problem. Minds and $\mathrm{Ma-}$ chines, 17(4), 369-389.

Grunwald, P. D., \& Vitanyi, P. M. 2008. Algorithmic Information Theory. In: Adriaans, P., \& van Benthem, J. (eds), Handbook on the Philosophy of Information. North Holland.

Haken, H. 1993a. Advanced Synergetics: Instability Hierarchies of Self-Organizing Systems and Devices. 3rd. edn. Springer, Berlin.

Haken, H. 1993b. Synergetics: An Introduction. 3rd edn. Springer, Berlin.

Haken, H. 2000. Information and Self-Organization: A Macroscopic Approach to Complex Systems. 2nd. edn. Springer.

Hinrichsen, D., \& Pritchard, A. J. 2005. Mathematical Systems Theory I - Modelling, State Space Analysis, Stability and Robustness. Springer.

Hirsch, M. W., Smale, S., \& Devaney, R. L. 2004. Differential Equations, Dynamical Systems, and an Introduction to Chaos. Academic Press.

Israel, D. J., \& Perry, J. R. 1990. What is Information? In: Hanson, P. (ed), Information, Language and Cognition: Vancouver Studies in Cognitive Science, vol. I. University of British Columbia Press.

Ivancevic, V. G., \& Ivancevic, T. T. 2008. Complex Nonlinearity: Chaos, Phase Transitions, Topology Change and Path Integrals. Understanding Com- plex Systems. Springer.

Jianbo Gao, Yinhe Cao, W.-w. T., \& Hu, J. 2007. Multiscale Analysis of Complex Time Series: Integration Of Chaos And Random Fractal Theory, And Beyond. Wiley-Interscience.

Katok, A., \& Hasselblatt, B. 1996. Introduction to the modern theory of dynamical systems. Cambridge.

Kelso, J. A. S. 1995. Dynamic Patterns: The SelfOrganization of Brain and Behavior. MIT Press.

Ladyman, J., Ross, D., Spurrett, D., \& Collier, J. 2007. Every Thing Must Go: Metaphysics Naturalized. Oxford.

Levine, W. S. (ed). 1996. The Control Handbook. New York: CRC Press.

Li, M., \& Vitanyi, P. 1997. An Introduction to Kolmogorov Complexity and Its Applications. Springer.

MacKay, D. M. 1969a. Information, Mechanisms and Meaning. MIT Press.

MacKay, D. M. 1969b. Meaning and Mechanism. In: Information, Mechanism and Meaning. MIT Press.

MacKay, D. M. 1969c. The Place of 'Meaning' in the Theory of Information. In: Information, Mechamnism and Meaning. MIT Press.

Maturana, H. R., \& Varela, F. J. 1980. Autopoiesis and Cognition: The Realization of the Living. Springer.

Millikan, R. 1987. Language, Thought, and Other Biological Categories: New Foundations for Realism. MIT Press.

Millikan, R. 1995. White Queen Psychology and Other Essays for Alice. MIT Press.

Millikan, R. 2006. Varieties of Meaning: The 2002 Jean Nicod Lectures. MIT Press.

Morris, C. W. 1938. Foundations of the Theory of Signs. University of Chicago Press.

Nauta, D. 1970. The Meaning of Information. Mouton.

Newell, A., \& Simon, H. 1981. Computer science as empirical enquiry. In: Hougeland, J. (ed), Mind design. MIT Press.

Peirce, C. 1940. Logic as Semiotic: The Theory of Signs. In: The Philosophy of Peirce: Selected Writings. Routledge and Kegan Paul.

Putnam, H. 1975. The Meaning of 'Meaing'. In: Gunderson, K. (ed), Language, Mind and Knowlege. Minnesota Studies in the Philosophy of Scince, no. VII. Unversity of Minnesota Press.

Seligmen, J. 1991. Physical Situations and Information Flow. In: Robin Cooper, Kuniaki Mukai, J. B. J. P. (ed), Situation Theory and its Applications, vol. 2. CSLI.

Sethna, J. P. 2009. Statistical Mechanics: Entropy, Order Parameters, And Complexity. Clarendon Press.

Shannon, C. 1948. The Mathematical Theory of Communication. Bell Systems Technical Journal. 
Thelen, E., \& Smith, L. 1994. Dynamics system approach to the development of cognition and action. MIT Press.

Turchin, V. 1990. Cybernetics and Philosophy. Pages 61-74 of: Geyer, F. (ed), The Cybernetics of Complex Systems. Intersystems, Salinas, California.

Vakarelov, O. forthcoming. The Cognitive Agent: Overcoming informational limits. Philosophical Psychology.

van Gelder., T. 1998. The dynamical hypothesis in cognitive science. Behavioral and Brain Sciences, 21, 615-628.

Varela, F. 2000. El fenomeno de la vida. Santiago de Chile: Dolmen Ediciones.

Varela, F. J., Thompson, E. T., \& Rosch, E. 1992. The Embodied Mind. MIT Press.

von Uexküll, J. 1909. Unwelt und Innenwelt der Tierre. Springer, Berlin.

von Uexküll, J. 1932. Streifziige durch die Umwelten von Tieren und Menschen. Springer, Berlin.

von Uexküll, J. 1982. The Theory of Meaning. Semiotica, 42(1), 25-82.

Weaver, W., \& Shannon, C. E. 1963. The Mathematical Theory of Communication. Univ. of Illinois Press.

Weber, A., \& Varela, F. 2002. Life after Kant: Natural purposes and the autopoietic foundations of biological individuality. Phenomenology and the Cognitive Sciences, 1(2), 97-125.

Wiener, N. 1965. Cybernetics: or the Control and Communication in the Animal and the Machine. MIT Press.

Williams, P., \& Beer, R. Forthcoming. Information dynamics of evolved agents. In: From Animals to Animats 11: Proceedings of the 11th International Conference on Simulation of Adaptive Behavior. 